\title{
Isolation of Microorganisms According to Burned Body Surface: Experience in a Reference Center in Mexico City
}

\author{
Carlos I. Navarro-Delgadillo, Jaime Aron García-Espinoza, Daniel De Luna-Gallardo, \\ José Martínez-López, Cuahutemoc Márquez-Espriella, Marco Cuervo-Vergara, \\ Rodrigo Dávila-Díaz
}

Hospital Central Sur de Alta Especialidad PEMEX, Ciudad de México, Mexico

Email: c_illich@hotmail.com

How to cite this paper: Navarro-Delgadillo, C.I., García-Espinoza, J.A., De LunaGallardo, D., Martínez-López, J., MárquezEspriella, C., Cuervo-Vergara, M. and Dávila-Díaz, R. (2021) Isolation of Microorganisms According to Burned Body Surface: Experience in a Reference Center in Mexico City. Journal of Biosciences and Medicines, 9, 106-113. https://doi.org/10.4236/jbm.2021.96010

Received: May 7, 2021

Accepted: June 15, 2021

Published: June 18, 2021

Copyright $\odot 2021$ by author(s) and Scientific Research Publishing Inc. This work is licensed under the Creative Commons Attribution International License (CC BY 4.0).

http://creativecommons.org/licenses/by/4.0/

\section{(c) (i) Open Access}

\begin{abstract}
Background: Burns is one of the most common and severe forms of trauma, as they cause an alteration in the protective barrier exerted by the skin. The objective of this work is to describe the bacterial pathogens that cause infections in the different body segments as well as to know the susceptibility in patients with burns during a period of two years. Methods: This study was conducted over a period of 2 years, January 2017 to January 2019. The analysis of the isolated microbiological agents was obtained from culture samples in different anatomic areas. The variables were measured in the general population and also by groups, considering: age, sex, TBSA with Wallace's rule of nine, anatomic area affected, type of burn, depth of burn, microorganism reported in cultures, antibiotics empirically administered and mortality rate. Results: Regardless of the anatomical segment studied, the most frequently isolated microorganism was Pseudomonas aeruginosa. Conclusions: Identification of the microorganisms that cause infection reduces the hospital stay, as well as the admission to the ICU. A fundamental research guideline is to pay more attention to the post-burn consequences, and the sequelae and functional limitations that it entails, since few since few studies focus on this aspect.
\end{abstract}

\section{Keywords}

Burn Injury, Hospital Stay, Specialized Burn Unit, Pseudomona

\section{Introduction}

Burns is one of the most common and severe forms of trauma, as they cause an alteration in the protective barrier exerted by the skin. These occur in different 
degrees of extension, depth and location, which is directly related to the severity of the burn [1].

Burns is the most devastating form of trauma. Records and data on burns and their treatment date back 3500 years, and their management has represented a changing therapeutic challenge. However, the advances that revolutionized its treatment were developed in the United States in the 18th century, when Allen et al. observed a decrease in the incidence of infections in burn patients when applying silver $1 \%$ sulfadiazine topical cream [2].

It is estimated that 11 million burn patients are treated worldwide each year, being reported around 300,000 total deaths, of which $90 \%$ occurs in developing countries [3]. In Mexico, 10 years ago, burns ranked seventeenth in incidence of trauma, affecting 129,779 people, predominantly men between 24 and 40 years of age. Their impact is due to the complex response they trigger and the cost of care, estimating an approximate expense between 1500.00 and 50,000.00 for moderate burns and up to 2 million dollars for severe burns [4].

Recently, several randomized controlled trials have evaluated the use of topical and systemic prophylactic antibiotics, demonstrating that its use did not show benefits when administered on admission or perioperatively; concluding that the available evidence is limited and, in general, does not demonstrate that antibiotic prophylaxis reduces the risk of burn infection, opportunistic infections (Ols) or mortality associated with the infection [5] [6].

People with severe burns are at increased risk of developing local and systemic infections. Currently, due to advances in initial fluid resuscitation, the presence of infection and its associated complications constitute the main cause of morbidity and mortality in this type of patient. Early exposure to a virulent pathogen after injury can rapidly progress to soft tissue infection and complicate with systemic inflammatory responses and even septic shock. As the levels of bacterial growth increase due to colonization of wounds, the incidence of invasion of viable tissues and the systemic inflammatory response increases, causing sepsis [7].

In recent decades, Gram-negative microorganisms have emerged as the most common etiological agents causing invasive infections due to their high dissemination capacity and antimicrobial resistance patterns. For this reason, it is extremely important to know the in-hospital microbiological epidemiology, in order to take preventive measures associated with these infections and to establish empirical and targeted treatment for each of them [8] [9].

The importance of taking a culture sample in burned patients lies in the need to be able to face this pathology in an integral way, trying to reduce the risks of a severe infection, the need for intensive therapy management, reduce the days of hospital stay, as well as reducing hospital expenses, by not having a first-level microbiology service, we must wait for the development of microorganisms, which on average vary from 5 to 7 days, so we know the most followed organisms in our population, could streamline decision making.

The objective of this work is to describe the bacterial pathogens that cause in- 
fections in the different body segments as well as to know the susceptibility in patients with burns during a period of two years in the Burn Center of the "Hospital Central Sur de Petróleos Mexicanos" in Mexico City.

\section{Materials and Methods}

A retrospective, descriptive and cross-sectional study was carried out including patients who met the criteria for total body surface area (TBSA) greater than $10 \%$ admitted to our hospital, all patients with at least one positive culture taken at admission, after a week of hospitalization. This study was conducted over a period of 2 years, January 2017 to January 2019.

The analysis of the isolated microbiological agents was obtained from culture samples in different anatomic areas.

The following variables were measured in the general population and also by groups, considering: age, sex, TBSA with Wallace's rule of nine, anatomic area affected, type of burn, depth of burn, microorganism reported in cultures, antibiotics empirically administered and mortality rate.

Patients with signs of bloodstream infections, TBSA less than $10 \%$, chemical burns and those who did not have all the study variables in their electronic medical record were excluded, in order to eliminate possible biases.

The microbiological determination was carried out by taking cultures with a dry swab in the different affected anatomical areas (using a swab for each segment), which were divided as follows: head and neck, thorax/back, abdomen, upper limb, lower limb and genitals. The microorganisms were identified by the microbiology service of the same hospital, repeating the sampling after 7 days of hospitalization in order to direct the empirical therapy used.

The culture swabs used were COPAN brand (Zacatecas, Mexico); swab with a tip covered with short nylon fibers that are arranged in a perpendicular way, for efficiency in the sample collection process and with fast and automatic elution, each plate was incubated at 35 degrees centigrade, under aerobic conditions. The microorganisms were identified with the VITEK system (Biomérieux, France) and the susceptibility was carried out according to the guidelines of the Clinical \& Laboratory Standards Institute (2017), obtaining a preliminary development at 72 hours and a definitive result at 7 days.

\section{Results}

We had a total of thirty-eight patients, who were divided into two groups. $80 \%$ were male and $20 \%$ female, the mean age was 41 years with an age range of 27 to 55 years. $80 \%$ of the patients had TBSA greater than $30 \%$, the average TBSA was $32.5 \%$ and the interquartile range was $20 \%$ to $50 \%$ (Figure 1 ).

It was observed that $30 \%$ of the patients, at the time of admission, did not show colonization of microorganisms in the first taking of cultures in burned surface areas. Seven days after their hospital stay, a second culture taking was made, showing development of the following organisms: Pseudomonas aerugi- 
nosa, Candida albicans, Enterobacter cloacae in order of frequency (Figure 2). Regardless of the anatomical segment studied, the most frequently isolated microorganism was Pseudomonas aeruginosa (Table 1).

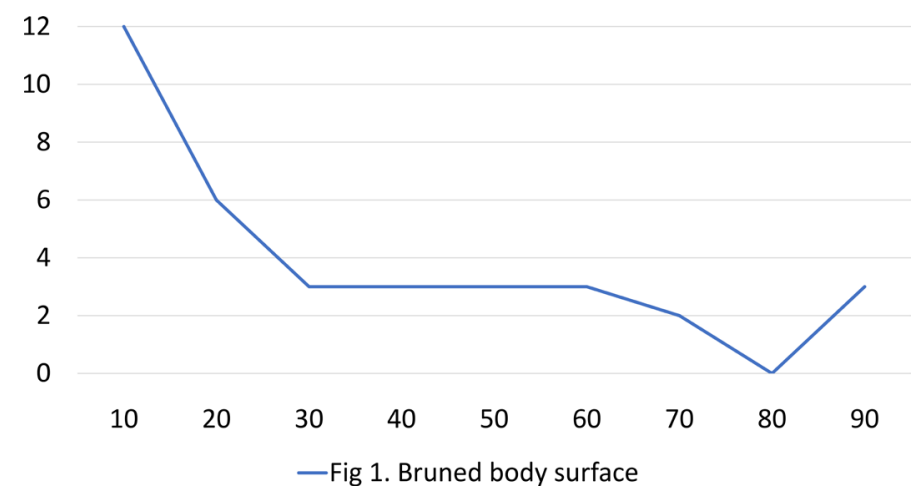

Figure 1. Bruned body surface.

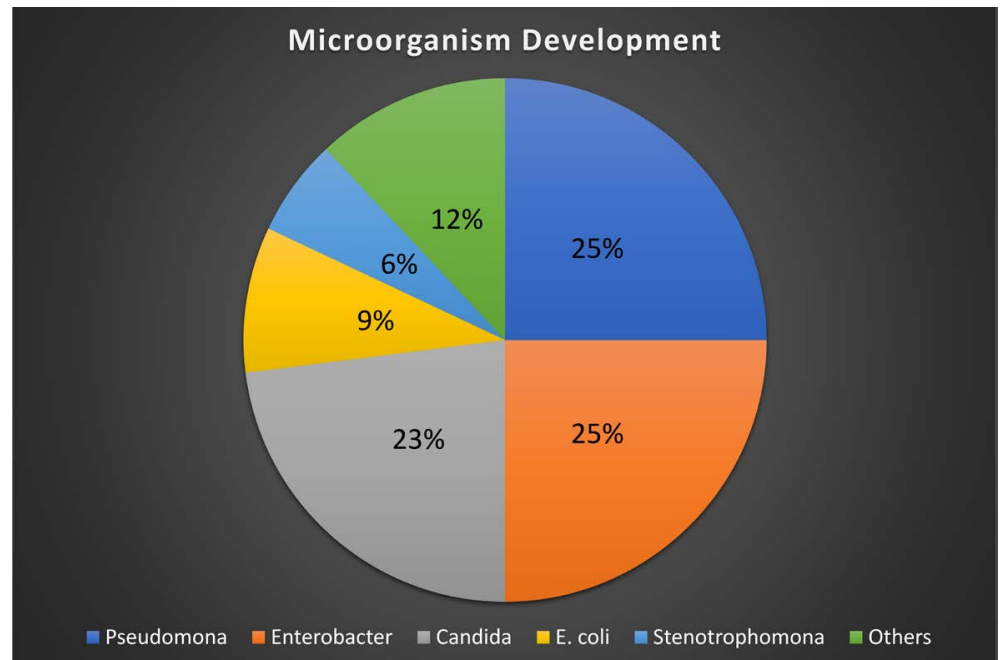

Figure 2. Microorganism isolated in culture.

Table 1. Isolated microorganisms.

\begin{tabular}{|c|c|c|c|c|c|c|c|c|c|c|c|}
\hline Columna1 & 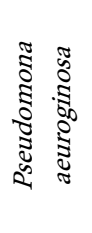 & 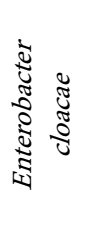 & 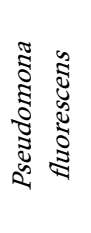 & 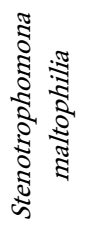 & 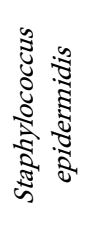 & 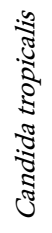 & 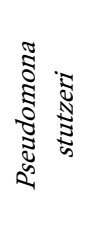 & 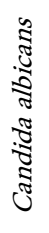 & 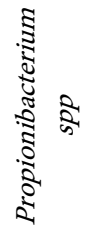 & 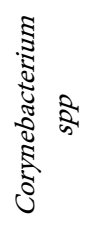 & 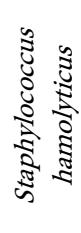 \\
\hline Head and Neck & 4 & 2 & 1 & 1 & 1 & 0 & 0 & 0 & 0 & 0 & 0 \\
\hline Thorax & 5 & 1 & 1 & 1 & 0 & 1 & 1 & 0 & 0 & 0 & 0 \\
\hline Abdomen & 2 & 0 & 1 & 1 & 0 & 0 & 0 & 0 & 0 & 0 & 0 \\
\hline Upper extremity & 5 & 0 & 1 & 1 & 0 & 1 & 0 & 4 & 0 & 0 & 0 \\
\hline Lower limb & 5 & 0 & 1 & 1 & 0 & 2 & 1 & 0 & 1 & 1 & 0 \\
\hline Genitals & 3 & 0 & 0 & 1 & 0 & 0 & 0 & 0 & 0 & 0 & 1 \\
\hline
\end{tabular}

Microorganisms in isolated in different anatomical segments. 
$96 \%$ of the patients received empirical antibiotic therapy, within which $64 \%$ are modified to targeted antibiotic therapy after receiving the results of the culture and antibiogram. Third-generation cephalosporins were the most widely administered antibiotic, followed by carbapenems, and finally combinations of these drugs. The most commonly used combination was a broad-spectrum penicillin plus an anaerobicide.

\section{Discussion}

An infection in burned patient conditions causes an important inflammatory response in the body, which develop various systemic symptoms, such as tachycardia, hypotension and fever. The importance of bacterial identification lies in establishing empirical antibiotic treatment and subsequently directed treatment to the identified microorganism according to its susceptibility [10]. Several studies have been carried out in which it has been observed that patients with invasive infection who received inadequate treatment according to the type of microorganism isolated, they had more complications and higher rate of mortality [11].

Since our Hospital is a referral burn center, our patients mainly have full thickness burns, burns in special areas (genitals, face and hands) or more than $10 \%$ of TSBA. A significant percentage of patients have had concomitant trauma and airway injuries, which is why they have worse prognosis and greater complexity in their management. In our population, the most affected anatomical segment was the upper extremities, followed by the head and neck.

In Mexico, the average cost per episode of in-hospital infection has been reported to be $\$ 4200$ to $\$ 8990$ [12]. In recent decades, a microbiological change has been observed in the bacteria involved in infectious processes towards an increase in Gram-negative bacilli. P. aeruginosa is a ubiquitous opportunistic microorganism, with a great capacity to adapt to the environment. In several previous reports this bacillus has been located as one of the main microorganisms causing nosocomial infections [13].

Previous studies carried out in our hospital showed that patients who developed Pseudomonas in cultures had a longer ICU and hospital stay, significantly longer than those who developed other microorganism, and also required a greater number of surgeries [14]. Other studies have also shown that Pseudomonas infection is correlated with increased resistance to antibiotics, prolonged hospital stay, and a higher mortality [15] [16] [17].

On the other hand, $C$. albicans was the second isolated microorganism, followed by enterobacteria. The before mentioned microorganisms are associated with the presence of bacterial resistance and secondarily with lack of therapeutic options for its eradication.

Unfortunately, the main isolated microorganisms are those that have been reported in other studies, in addition to an increase in antibiotic resistance. For its control, personal protection measures for health personnel should be pro- 
moted, improving clinical-surgical practices, with special emphasis on hand washing and the use of an antimicrobial control policy, as recommended globally [18].

As a retrospective study, it had certain limitations, not being able to define whether other variables, such as type and number of surgeries, the dressings used or some other invasive procedures, could be related to the development of the infection. However, the data gave us a broad view of the bacterial microorganisms that are associated with post-burn patients, which shows possible alternatives in the treatment and prevention of infections in these types of patients.

Most of the patients received empirical antibiotics, and more than 50\% underwent modifications of this treatment after the culture results were obtained. We believe that this maneuver improved the prognosis of severely affected patients. Another factor that we believe favored recovery and resulted in a mortality rate similar to that of first world countries, is that throughout the hospital stay there was close 24-hour surveillance by a whole group of plastic surgeons, plastic surgical residents, intensive care doctors, nurses, nutritionists and rehabilitators.

\section{Conclusions}

The correct care of burn patients is one of the most complex challenges in trauma management, since it requires multidisciplinary intervention, highly specialized management, and represents a high socioeconomic impact. Identification of the microorganisms that cause infection reduces the hospital stay, as well as the admission to the ICU.

It is necessary to emphasize preventive medicine in order to reduce the incidence of this type of accidents and therefore burn injuries; the development of prevention programs at home, initial management by paramedics, an effective health system that includes referral centers and units specialized in burn patient care, is essential to increase their survival.

In order to offer a greater recovery, with less hospitalization than that reported in the literature, it is necessary to identify microorganisms that cause infections on burned body surfaces, in order to provide targeted therapy, reducing bacterial resistance, days of hospital stay, need for ICU management and hospital costs.

A fundamental research guideline is to pay more attention to the post-burn consequences, and the sequelae and functional limitations that it entails, since few since few studies focus on this aspect.

\section{Gratitude}

We dedicate this work to all patients and their families who suffer these types of injuries in our society.

\section{Conflicts of Interest}

The authors declare no conflicts of interest regarding the publication of this pa- 
per.

\section{References}

[1] Church, D., Elsayed, S., Reid, O., Winston, B. and Lindsay, R. (2006) Burn Wound Infections. Clinical Microbiology Reviews, 19, 403-434. https://doi.org/10.1128/CMR.19.2.403-434.2006

[2] Hettiaratchy, S. and Dziewulski, P. (2004) ABC of Burns: Pathophysiology and Types of Burns [Published Correction Appears in BMJ. 2004 Jul 17; 329(7458): 148]. BMJ, 328, 1427-1429. https://doi.org/10.1136/bmj.328.7453.1427

[3] Peck, M.D. (2011) Epidemiology of Burns throughout the World. Part I: Distribution and Risk Factors. Burns, 37, 1087-1100.

https://doi.org/10.1016/j.burns.2011.06.005

[4] Moctezuma-Paz, L., Paez-Franco, I., Jimenez-Gonzalez, S., Sanchez-Flores, A.Y., et al. (2015) Epidemiologia de las quemaduras en Mexico. Revista de Especialidades Médico-Quirúrgicas, 20, 78-82.

[5] Barajas-Nava, L.A., López-Alcalde, J., Roqué i Figuls, M., Solà, I. and Bonfill Cosp, X. (2013) Antibiotic Prophylaxis for Preventing Burn Wound Infection. Cochrane Database of Systematic Reviews, No. 6, CD008738. https://doi.org/10.1002/14651858.CD008738.pub2

[6] Navarro-Delgadillo, C.I., et al. (2020) Use of the Abbreviated Burn Severity Index (ABSI) as a Severity Scale in a Burn Unit in Mexico: A 2-Year Experience. European Journal of Plastic Surgery, 44, 111-116. https://doi.org/10.1007/s00238-020-01724-X

[7] Branski, L.K., Al-Mousawi, A., Rivero, H., Jeschke, M.G., Sanford, A.P. and Herndon, D.N. (2009) Emerging Infections in Burns. Surgical Infections, 10, 389-397. https://doi.org/10.1089/sur.2009.024

[8] Martin, G.S., Mannino, D.M., Eaton, S. and Moss, M. (2003) The Epidemiology of Sepsis in the United States from 1979 through 2000. The New England Journal of Medicine, 348, 1546-1554. https://doi.org/10.1056/NEJMoa022139

[9] Rennie, R.P., Jones, R.N., Mutnick, A.H. and SENTRY Program Study Group (North America) (2003) Occurrence and Antimicrobial Susceptibility Patterns of Pathogens Isolated from Skin and Soft Tissue Infections: Report from the SENTRY Antimicrobial Surveillance Program (United States and Canada, 2000). Diagnostic Microbiology and Infectious Disease, 45, 287-293. https://doi.org/10.1016/S0732-8893(02)00543-6

[10] Kumar, A., Ellis, P., Arabi, Y., et al. (2009) Initiation of Inappropriate Antimicrobial Therapy Results in a Fivefold Reduction of Survival in Human Septic Shock. Chest, 136, 1237-1248. https://doi.org/10.1378/chest.09-0087

[11] Kumar, A., Roberts, D., Wood, K.E., et al. (2006) Duration of Hypotension before Initiation of Effective Antimicrobial Therapy Is the Critical Determinant of Survival in Human Septic Shock. Critical Care Medicine, 34, 1589-1596. https://doi.org/10.1097/01.CCM.0000217961.75225.E9

[12] Nava, A.R., Gonzalez, G.R. and Rosas, A.T. (2012) Infecciones Hospitalarias. Reviste digital Universitaria., 13.

[13] Chong, S.J., Ahmed, S., Tay, J.M., Song, C. and Tan, T.T. (2011) 5 Year Analysis of Bacteriology Culture in a Tropical Burns ICU. Burns, 37, 1349-1353.

https://doi.org/10.1016/j.burns.2011.07.020

[14] Garcia-Espinoza, J.A., Navarro-Delgadillo, C.I., Costa-Dulche, A. and Marquez-Espriela, C. (2019) Epidemiology of Burn Injuries: 2 Years' Experience in a 
Specialized Hospital in Mexico City. Annals of Burns and Fire Disasters, 32, 261-266.

[15] Gong, Y.L., Chen, J., Liu, C.J., et al. (2014) Comparison of Pathogens and Antibiotic Resistance of Burn Patients in the Burn ICU or in the Common Burn Ward. Burns, 40, 402-407. https://doi.org/10.1016/j.burns.2013.07.010

[16] Panghal, M., Singh, K., Kadyan, S., Chaudary, U. and Yadav, J.P. (2015) The Analysis of Distribution of Multidrug Resistant Pseudomonas and Bacillus Species from Burn Patients and Burn Ward Environment. Burns, 41, 812-819.

https://doi.org/10.1016/j.burns.2014.10.014

[17] Issler-Fisher, A.C., Fakin, R.M., Fisher, O.M., et al. (2016) Microbiological Findings in Burn Patients Treated in a General versus a Designated Intensive Care Unit: Effect on Length of Stay. Burns, 42, 1805-1818.

https://doi.org/10.1016/j.burns.2016.06.019

[18] Croft, L.D., Liquori, M., Ladd, J., et al. (2015) The Effect of Contact Precautions on Frequency of Hospital Adverse Events. Infection Control \& Hospital Epidemiology, 36, 1268-1274. https://doi.org/10.1017/ice.2015.192 